\title{
Application opportunities of geographic information systems analysis to support achievement of the UNAIDS 90-90-90 targets in South Africa
}

\author{
R R Lilian, ${ }^{1}$ MSc (Med); C J Grobbelaar, ${ }^{1}$ MB ChB, MFamMed; T Hurter, ${ }^{1}$ MPH; J A McIntyre, ${ }^{1,2}$ MB ChB, FRCOG; H E Struthers, ${ }^{1,3}$ PhD; \\ R P H Peters, ${ }^{1}$ MD, PhD, DLSHTM, Dip HIV Man (SA) \\ ${ }^{1}$ Anova Health Institute, Johannesburg and Cape Winelands, South Africa \\ ${ }^{2}$ School of Public Health and Family Medicine, Faculty of Health Sciences, University of Cape Town, South Africa \\ ${ }^{3}$ Division of Infectious Diseases and HIV Medicine, Department of Medicine, Faculty of Health Sciences, University of Cape Town, South Africa
}

Corresponding author: R P H Peters (peters@anovahealth.co.za)

\begin{abstract}
In an effort to achieve control of the HIV epidemic, 90-90-90 targets have been proposed whereby $90 \%$ of the HIV-infected population should know their status, $90 \%$ of those diagnosed should be receiving antiretroviral therapy, and $90 \%$ of those on treatment should be virologically suppressed. In this article we present approaches for using relatively simple geographic information systems (GIS) analyses of routinely available data to support HIV programme management towards achieving the 90-90-90 targets, with a focus on South Africa (SA) and other high-prevalence settings in low- and middle-income countries. We present programme-level GIS applications to map aggregated health data and individual-level applications to track distinct patients. We illustrate these applications using data from City of Johannesburg Region D, demonstrating that GIS has great potential to guide HIV programme operations and assist in achieving the 90-90-90 targets in SA.
\end{abstract}

S Afr Med J 2017;107(12):1065-1071. DOI:10.7196/SAMJ.2017.v107i12.12666

Geographic information systems (GIS) analysis refers to the capture, management, evaluation and visualisation of geographically referenced data, although the term can generally be applied to any investigation that incorporates a location variable. ${ }^{[1]}$ This can range from simple maps that provide information about point locations, for example facilities, to complex spatial statistical analyses. ${ }^{[2]}$ GIS is an important tool to advance public health, as many aspects of health and illness have a geographical or spatial component. ${ }^{[2]}$ GIS applications may include mapping of disease distribution, assessing patterns of health interventions such as immunisations, investigating spatial and temporal trends, mapping variations in risk factors and forecasting epidemics. ${ }^{[2]}$

One of the major health challenges facing South Africa (SA) and other low- and middle-income countries (LMICs), particularly in sub-Saharan Africa, is the HIV epidemic. In an effort to achieve epidemic control, the Joint United Nations Programme on HIV/ AIDS (UNAIDS) has proposed ambitious 90-90-90 targets to be achieved by 2020 , whereby $90 \%$ of people infected with HIV will have received a diagnosis, $90 \%$ of those diagnosed will be receiving sustained antiretroviral therapy (ART), and $90 \%$ of those on treatment will be virally suppressed.$^{[3]}$ GIS analyses, often involving complex spatial statistics, have been used to investigate various aspects of the HIV epidemic in a number of countries including SA, for example to examine the distribution of HIV infection, including hot-spots or clusters of high or low prevalence; ${ }^{[4-17]}$ to assess the spatial distribution of factors contributing to HIV infection; ${ }^{[4,14,18]}$ to describe the spatial distribution of the HIV care continuum (HIV testing, uptake of ART, viral suppression) and the factors that affect provision of this care, including location of services; ${ }^{[19-27]}$ to investigate HIV infection, prevention services and location/density of key populations, including injection drug users, men who have sex with men and sex workers; ${ }^{[28-33]}$ to guide or map prevention efforts such as male circumcision or condom distribution ${ }^{[34,35]}$ to understand patterns of HIV knowledge; ${ }^{[36]}$ and to examine distribution of funding for HIV services. ${ }^{[37]}$ Although some of these applications can inform efforts towards achieving the 90-90-90 objectives, to our knowledge no systematic approach has been presented to define the role of GIS in achieving these targets. Furthermore, GIS analyses may be of limited use in LMICs if complex analyses are performed that require highly skilled personnel or if the analyses primarily draw on research data, with no link to routine, programme data that can be used to inform programme implementation.

In this article we present approaches for using relatively simple GIS analyses of routinely available data to support HIV programme management towards achieving the 90-90-90 targets, with a focus on SA and other high-prevalence settings in LMICs. Using data from City of Johannesburg (CoJ) Region D, we present applications at a programme level to map aggregated health data and at an individual level to track distinct patients, both of which can guide efforts towards achieving the 90-90-90 targets.

\section{The firest 90: Diagnosis of $90 \%$ of} people infected with HIV

GIS can be used to guide progress towards the first 90 by mapping population groups that require improved access to HIV testing services (HTS). For example, since a high number of HIV-infected males in SA remain undiagnosed, with low HTS uptake also recorded among men in other LMICs, ${ }^{[38,39]}$ visualising male population density by ward would be useful to identify specific areas with high concentrations of men. These areas, identified as wards 27, 30 and 45 in CoJ Region D (Fig. 1, A), could then be targeted for testing, for example, mobilising community health workers (CHWs) or mobile 
testing units to perform community-based testing after hours or over weekends when men, most of whom would be at work during routine clinic hours, would more likely be available for testing. Uptake of HIV testing has been shown to be particularly low among adolescent and young males aged 12 - 24 years, ${ }^{[40,41]}$ and a similar map showing population density of this target group can be generated to further focus testing in areas where this specific age group is more likely to be found (Fig. 1, B). A further use of GIS to guide progress towards the first 90 is mapping of high-risk populations to direct testing for higher yield. Explanatory variables associated with HIV prevalence, many of which have been mapped in SA and LMICs, ${ }^{[4,12,14,18]}$ can be simply visualised with pie charts to identify locations with populations at high risk of contracting HIV (Fig. 2). ${ }^{[14]}$ This may include populations with low socioeconomic status, multiple sexual partners or high rates of intergenerational sex. ${ }^{[14]}$ HTS resources could then be directed to these areas for potentially high-yield testing. Interestingly, in CoJ Region D, ward 40 appears to be a target area based on socioeconomic status (Fig. 2) as well as population density of adolescent and young males (Fig. 1, B), suggesting that focused efforts to provide HTS to this area may well assist in supporting progress toward the first 90 target. In addition, more complex GIS techniques have been used in several African settings to identify clusters or hotspots where HIV prevalence is significantly higher than in surrounding areas, ${ }^{[8,13,15]}$ which may also direct testing for higher yield by identifying locations where there are likely to be higher numbers of individuals living with HIV.

The application of GIS in the context of the first 90 would be largely at a programme level. Applications using individual-level data, for example plotting individuals who have undergone HIV testing, would only be of value with specific populations in small geographical areas and would therefore be of limited use in the context of the first 90 , which focuses on higher-level population screening.

\section{The second 90: 90\% of people with diagnosed HIV infection on ART}

Achieving the second $90 \%$ target by 2020 is likely to be a challenge in $\mathrm{SA},{ }^{[42]}$ and closing the treatment gap will require implementation of well-considered and innovative strategies. A compelling example of how GIS can guide this process is presented in Fig. 3, where GIS analysis of routine data is used to identify geographical areas with large gaps in ART

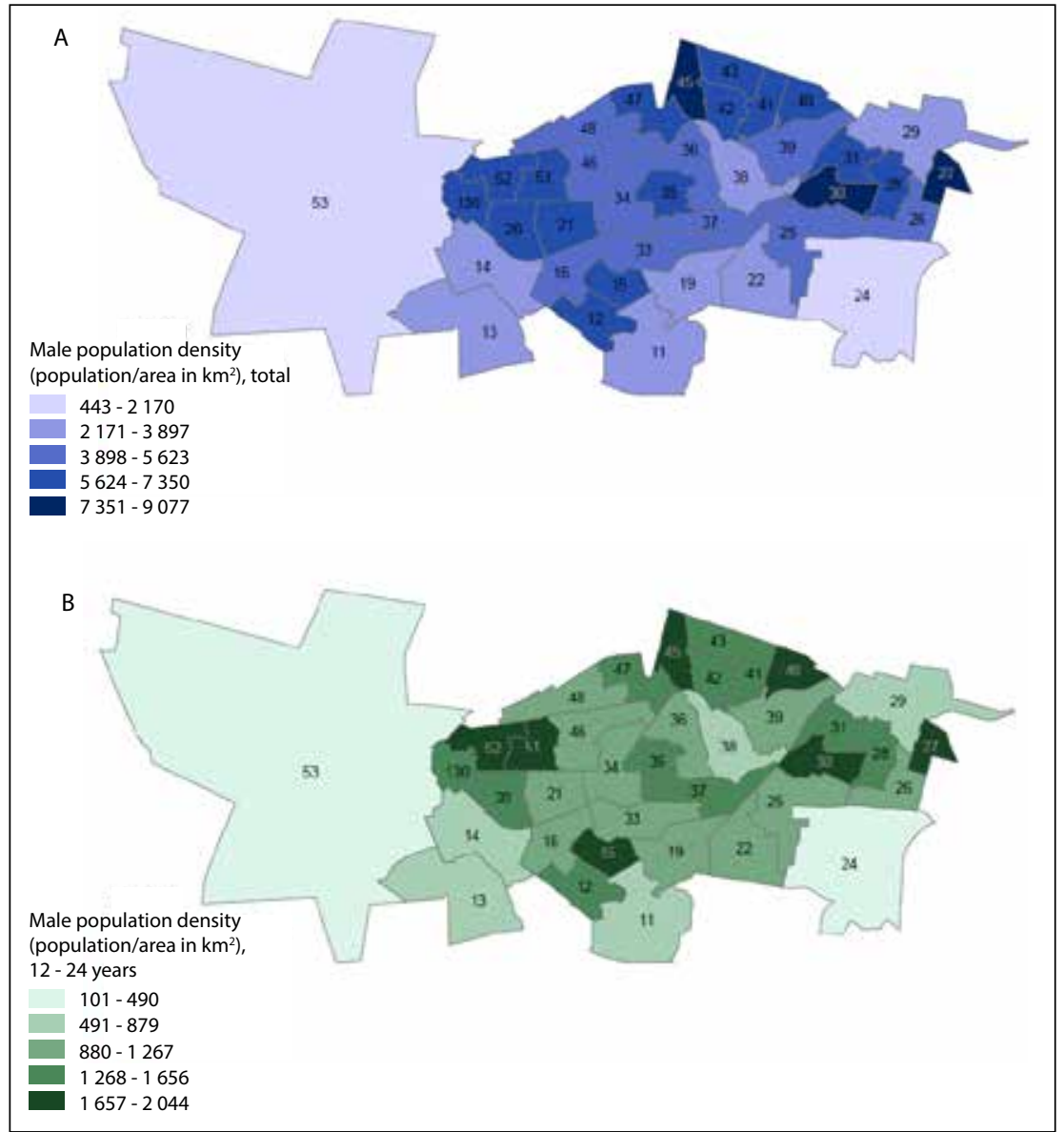

Fig. 1. Identifying the male target population for HIV testing in City of Johannesburg Region D. Population density (population/area in $\mathrm{km}^{2}$ ) by ward for (A) the total male population and (B) male adolescents and youth aged $12-24$ years (Census 2011 data $^{[43]}$ ) (the numbers on the figure are the ward numbers). Wards 27, 30 and 45 have a high density of total males and male youth. Four additional wards (15, 40, 51 and 52) also have a high density of younger males and can be targeted for HIV testing services to reach this target group.

coverage. The first step in this process is to estimate the HIV-infected population by age and gender. This is performed by multiplying population data, available from Statistics South Africa, ${ }^{[43]}$ by HIV prevalence data, which are publicly available from data sources such as the Thembisa model ${ }^{[44]}$ or, in the case of other LMICs, from antenatal surveillance or demographic and health surveys. The number of HIV-infected individuals receiving ART by age and gender, obtained from routine monitoring and evaluation (M\&E) data, can then be subtracted from the estimated HIV-infected population to calculate the programme gap, namely the number of HIV-infected individuals who are not receiving ART. By triangulating population and M\&E data in this way, the specific age and gender group with the greatest programme gap can be identified. Population and ART data for the target group in individual wards can then be overlaid and mapped to visualise wardlevel profiles (Fig. 3, B). These maps can be used to identify where the highest numbers of individuals who are missing from the ART programme are likely to be located, which in the case of CoJ Region D is wards 16, 33 and 37 for the male target group aged 30 - 39 years. Programme planners can thus strategically focus efforts to improve ART coverage in areas with the greatest need, including initiating communitybased awareness campaigns and expanding clinic-based services into the community through mobile clinics and alternative ART distribution points. Spatial patterns of ART coverage can be monitored over time by updating ART data in the heat maps on an ongoing basis, thereby tracking progress of interventions.

In view of the recommendation for universal test and treat, ${ }^{[45]}$ it is important to assess the proportion of the total HIVinfected population who are not receiving ART. This programme gap \% can be calculated by dividing the programme gap by the estimated HIV-infected population. 


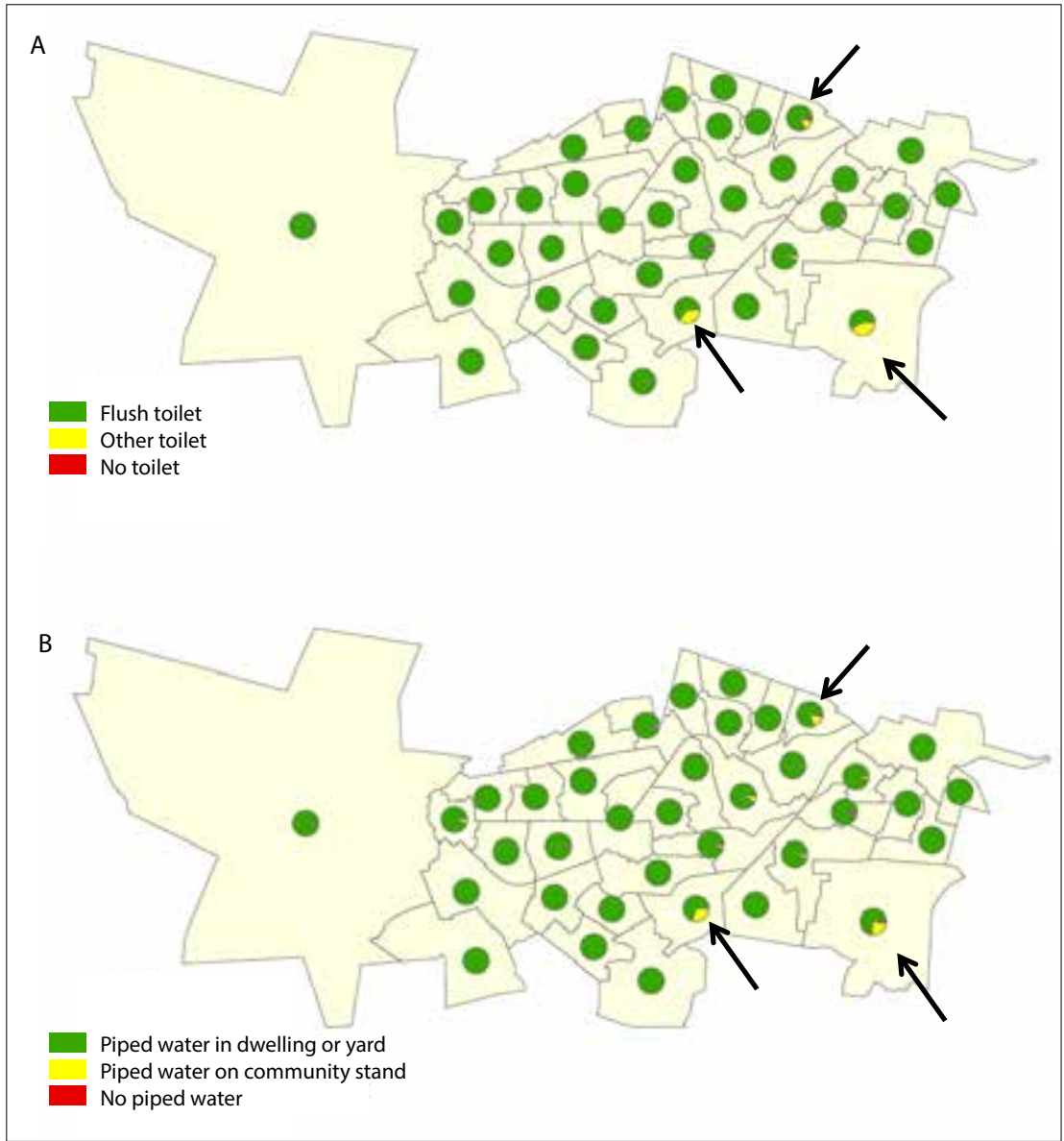

Fig. 2. Identifying potentially high-yield areas for HIV testing in City of Johannesburg Region D. Household access to (A) toilet facilities and (B) piped water by ward (Census 2011 data ${ }^{[43]}$ ). Wards 19, 24 and 40 (indicated by black arrows) have a lower proportion of households with flush toilets and in-dwelling or in-yard piped water and may be of lower socioeconomic status. Since low socioeconomic status is associated with an increased prevalence of $H I V,{ }^{[14]} \mathrm{HIV}$ testing services can be directed to these areas for potentially high-yield testing.

When overlaid with a heat map of the HIVinfected population, conclusions can be drawn regarding programme achievements in high-burden areas (Fig. 4). For example, in CoJ Region D, ward 11 has a large HIVinfected population among males aged 30 39 years with a very small programme gap $\%$, indicating that only a small proportion of the HIV-infected population is missing from the ART programme. This suggests that the programme has been well managed and supported in this area. In contrast, other wards with large numbers of HIVinfected individuals have a large programme gap \%, suggesting that these high-burden areas may benefit from further support. This GIS approach, which entails overlaying variables such as population estimates and programme performance, enables a nuanced approach to understanding performance of the ART programme in different areas.

GIS can further assist in supporting progress to the second $90 \%$ target by generating maps of population groups at high risk of attrition prior to ART initiation, such as males, adolescents and young adults. ${ }^{[46]}$ Interventions targeting these groups for ART initiation, such as community-based adherence clubs or services over the weekend or after work and school hours, can then be planned in areas with high proportions of these populations where there is a large gap in ART initiation. In addition, geographical barriers to uptake of treatment that may contribute to poor ART coverage can be identified using GIS by visualising service utilisation together with geographical variables such as location of roads, villages and healthcare facilities. Identifying geographical barriers to uptake of services allows deployment of innovative alternatives, for example motorbikes in remote areas ${ }^{[47]}$ or boats in island communities. ${ }^{[48]}$

In addition to programme-level applications, individual-level GIS analyses can also assist in guiding progress towards the second 90. Residences of individuals who test HIV-positive but are lost to treatment initiation can be mapped so that CHWs can perform home visits to link these individuals to care (sample map presented in Fig. 5; locations have been modified to protect patient confidentiality). This would not only be of clinical value for the individual patients, but would also serve to streamline CHW work and would inform programme planning by identifying areas with high numbers of individuals who are lost to initiation where communitydispensing points may be of benefit. In addition, maps can be used to identify HIVinfected individuals who live furthest from HIV clinics so that CHWs can proactively perform home visits to these high-risk individuals, as the odds of timeous ART access decrease with increasing distance from an HIV clinic, particularly for clinics located further from transportation hubs. ${ }^{[19,23]}$ The distance between patient locations and HIV clinics can be calculated using Google Earth, precluding the need for advanced computer skills or expensive software, ${ }^{[23]}$ and this approach would therefore be relatively easy to implement in LMICs.

\section{The third 90: 90\% of people on ART virally suppressed}

The third 90 refers to viral suppression rates in individuals receiving ART. However, in line with a 'cascade approach' to treatment targets, this objective should encompass wider efforts to improve longterm retention in care, with sustained HIV treatment, ongoing viral load monitoring and interventions to promote treatment adherence. ${ }^{[3]}$ Both retention in care and viral suppression rates can be calculated from routine M\&E data and the spatial distributions visualised with heat maps (as in Fig. 3, B) to identify areas that require targeted interventions. Using GIS one can visualise whether geographical areas of poor retention overlap with areas of poor viral suppression, which interestingly has not been found to be the case, suggesting that different factors may be responsible for poor outcomes at different steps of the HIV care cascade. ${ }^{[21]}$ GIS can also be used to visualise performance of individual healthcare facilities. In CoJ Region D, there are a number of facilities, located in the outer wards of the region, with viral suppression rates $<90 \%$ (Fig. 6). Targeting poor viral suppression rates in these areas may involve campaigns for viral load testing and addressing factors underlying poor ART adherence - for example, running public information campaigns addressing beliefs in divine healing, preference for traditional 
medicine and doubts regarding the benefits and safety of ART, while providing clinical training to address poor patient-provider relationships. ${ }^{[49-51]}$ It is also important for programme planning to identify areas with high rates of viral suppression, as individuals in these areas can be treated in the community to ease the burden on healthcare facilities. In addition to monitoring patterns of retention in care and viral suppression, GIS can be used to identify geographical barriers that may affect ART adherence, including infrastructure barriers such as inadequate transport. ${ }^{[52]}$ Poor access to healthcare facilities as a result of transport challenges highlights areas for communitybased interventions such as adherence clubs, alternative ART distribution points and deployment of mobile clinics.

Individual-level GIS analyses are highly beneficial in the context of the third 90 . Mapping households of individuals with poor adherence or those who have defaulted from ART care would enable CHWs to perform home visits to provide intensified adherence counselling and linkage back to care (Fig. 5). Using these maps, clusters of defaulters can then be identified. Where clusters are located near healthcare facilities, possible problems with facility services should be investigated and addressed. In addition, households with high-risk individuals can be mapped for pre-emptive home visits by CHWs. High-risk individuals for poor viral suppression include those who are economically deprived, ${ }^{[26]}$ while individuals living further from HIV clinics are at risk of poor adherence, as clinic distance is associated with missed clinic visits ${ }^{[27]}$ and transportation costs compromise access to care. ${ }^{[53]} \mathrm{CHWs}$ can also use area maps to talk to individuals about where best to locate alternative points for ART distribution that may facilitate improved adherence.

\section{Considerations for implementing GIS in routine settings}

As illustrated above for CoJ Region D and summarised in Table 1, GIS has many practical applications in support of programme operations working towards achieving the 90-90-90 targets. When implementing this technology in routine settings, it is firstly important to consider the choice of GIS software. Open-source, webbased GIS systems can reduce obstacles to end-user access and display a high degree of learnability, ${ }^{[54]}$ with web-based software that does not require specialised GIS skill having demonstrated potential for public health applications in LMICs. ${ }^{[55]}$ Affordability has

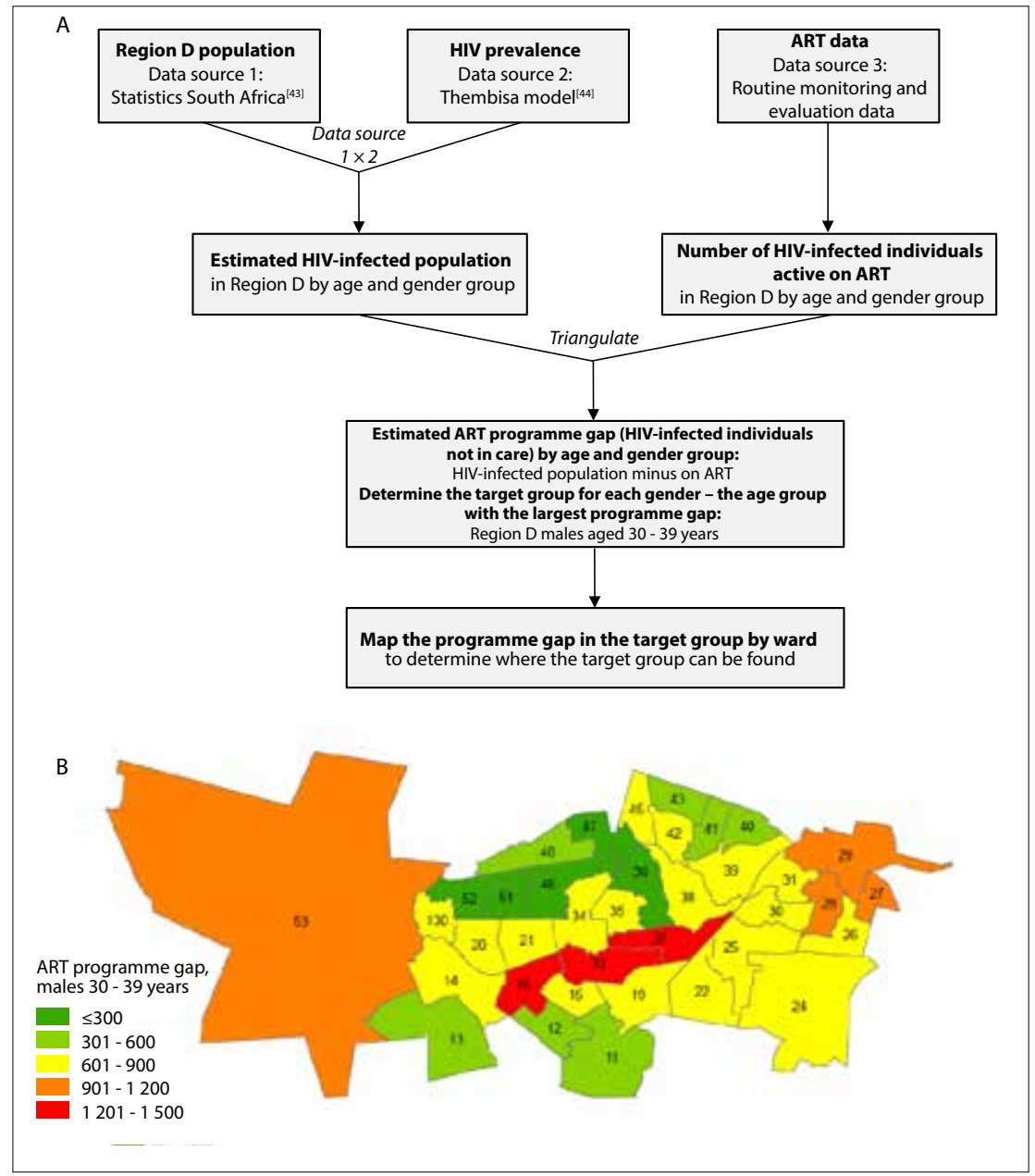

Fig. 3. Identifying wards with the highest gap in ART coverage in City of Johannesburg Region D. (A) Approach to calculating the ART programme gap in the HIV-infected population by ward. (B) Visual output of the ART programme gap by ward for the male target group aged 30 - 39 years clearly highlights that wards 16,33 and 37 (in red) have the greatest gap in service coverage (the numbers on the figure are the ward numbers). (ART = antiretroviral therapy.)

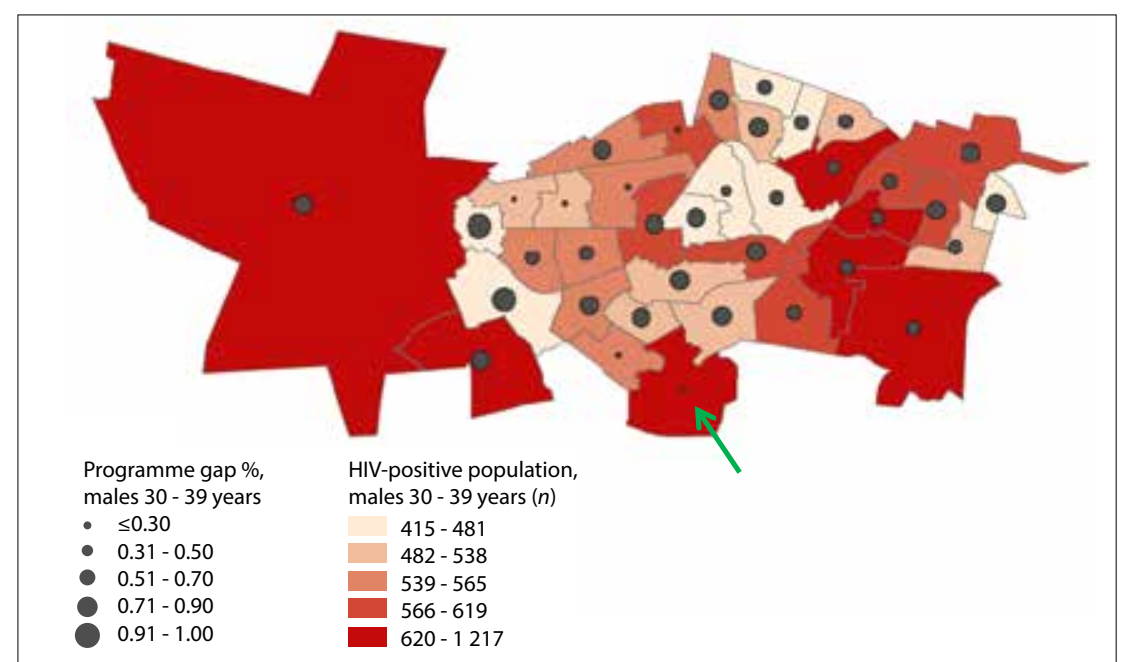

Fig. 4. Programme gap \% (proportion of the HIV-infected population not in care) and the HIV-infected population by ward among males aged 30 - 39 years in City of Johannesburg Region D. Ward 11 (indicated by a green arrow) has a large HIV-infected population with a very small programme gap $\%$, suggesting that the HIV programme has been well managed and supported in this high-burden area. In contrast, other wards in dark red with large numbers of HIV-infected individuals have a large programme gap \% and may benefit from further support. 
Table 1. Summary of potential applications of GIS to support achievement of the 90-90-90 targets

\begin{tabular}{|c|c|}
\hline & Programme-level support applications \\
\hline $\begin{array}{l}\text { First 90: diagnosis of } \\
90 \% \text { of people infected } \\
\text { with HIV }\end{array}$ & $\begin{array}{l}\text { - Map population density of target groups that require } \\
\text { improved access to HTS to identify areas with a high } \\
\text { density of these individuals for focussed HTS } \\
\text { - Map high-risk populations and/or risk factors } \\
\text { associated with HIV infection to identify potentially } \\
\text { high-yield areas for delivery of focused HTS } \\
\text { - Map clusters or hotspots of HIV prevalence to } \\
\text { identify areas where there are likely to be higher } \\
\text { numbers of HIV-infected individuals who can be } \\
\text { identified through HTS }\end{array}$ \\
\hline
\end{tabular}

Second 90: $90 \%$ of people with diagnosed HIV-infection on ART

Third 90: $90 \%$ of people on ART virally suppressed can be planned in areas with a high proportion of these populations

- Visualise geographical barriers to ART coverage that may contribute to poor treatment uptake, such as inadequate roads and long distances to clinics

- Visualise the spatial distribution of retention in ART care and viral suppression to identify areas with poor retention and/or suppression rates that require

\section{Individual-level support applications}

- Limited use - can map individuals who have undergone HIV testing to assess patterns of HTS uptake, but only in small geographical areas for specific populations

- Track patients who test HIV-positive but do not link to care - map residences of these individuals to facilitate home visits by CHWs

- Streamline CHW work by grouping visits to individuals who live in the same area

- Inform programme planning by identifying areas with high numbers of individuals lost to initiation where community-based dispensing points may be of benefit

- Plan proactive household visits by CHWs to HIVinfected individuals who live furthest from clinics to increase the likelihood of these high-risk individuals linking to care targeted interventions, such as viral load testing campaigns or addressing factors underlying poor ART adherence

- Identify areas with high rates of viral suppression where patients can be treated in the community to ease the burden on healthcare facilities

- Visualise facility-level viral suppression rates and assess whether facilities with poor performance cluster in certain areas

- Identify geographical barriers that may affect ART adherence, including infrastructure barriers
- Track patients who have defaulted from ART care or those with poor adherence - map residences of these individuals so that CHWs can perform home visits to provide intensified adherence counselling and re-linkage to ART care

- Identify ART facilities with potential service problems by identifying facilities around which there are clusters of individuals who have defaulted from care

- Plan pre-emptive home visits by CHWs to high-risk individuals who are likely to have compromised ART adherence or viral suppression

- Plan locations for alternative ART distribution points by using area maps to talk to individuals about where best to locate these services, thus facilitating improved adherence

GIS = geographic information systems; HTS = HIV testing service; ART = antiretroviral therapy; $\mathrm{CHW}=$ community health worker.

become less problematic over time, with the availability of free or inexpensive GIS software and easy mapping of point locations with inexpensive global positioning systems and/or mobile phones. ${ }^{[66,57]}$ Secondly, the poor quality of routine M\&E data, lack of unique patient identifiers and deficit of GIS data in some LMICs must be considered, in particular the availability of patient addresses for individual-level GIS applications. It is important to bear in mind that the definition of a patient address is multifaceted, as locations of work, sleep and family residence may vary, and that there may be no street names in rural settings for reporting patient addresses. Thirdly, it is essential to ensure confidentiality of individual-level patient data, particularly when using free web-based software. ${ }^{[34,58]}$ Finally, stakeholders may prove challenging if there is a lack of understanding regarding what GIS can deliver and how to interpret the outputs. Obtaining buy-in from stakeholders may also be difficult where there are concerns regarding transition from paper-based systems, misuse of information or political interference. ${ }^{[54,58]}$ It is important to address buy-in from the start and to provide adequate training for capacity development of local staff to ensure sustainable systems. ${ }^{[56]}$

\section{Conclusions}

GIS has great potential to assist in achieving the 90-90-90 targets in SA and other LMICs and is currently being under-utilised for this purpose. This article demonstrates opportunities for using easily accessible resources to generate GIS visualisations and underlines the important benefits GIS can have in guiding HIV programme operations, the principles of which can be applied to any infectious disease. GIS can be incorporated into routine programme planning by visualising the spatial distribution of programme implementation gaps and impact of interventions. Individual-level analyses are of clinical value for 


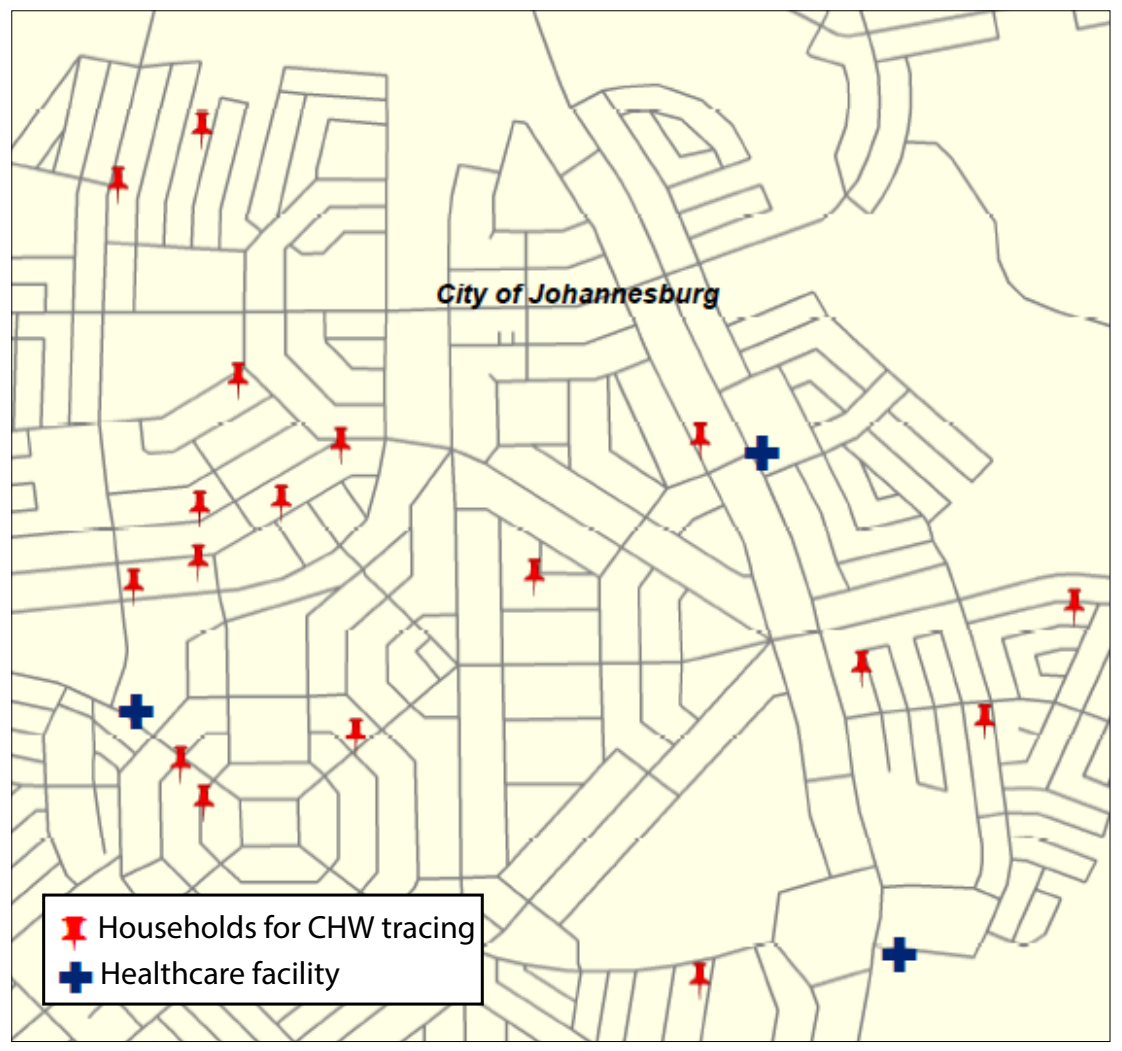

Fig. 5. Residences of HIV-infected individuals living in City of Johannesburg Region D who require home visits by CHWs (locations have been modified to protect patient confidentiality). Residences of $H I V$-infected individuals who are lost to treatment initiation or lost to follow-up from care can be georeferenced to generate maps that $\mathrm{CHWs}$ can use to perform home visits. (CHW = community health worker.)

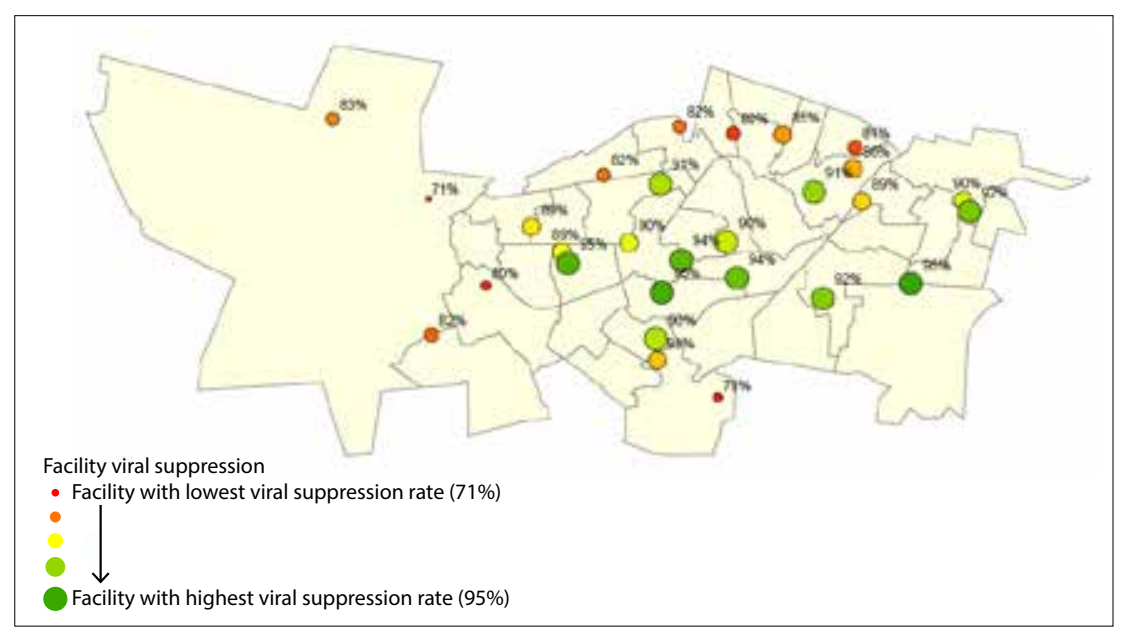

Fig. 6. Viral suppression rates at primary healthcare facilities in City of Johannesburg Region D. Facilities in red and orange with lower viral suppression rates, located around the outside of the region, should receive targeted interventions to improve progress towards the third $90 \%$ target.

patients and can also guide programme-level decisions. Interactive, dynamic systems using inexpensive GIS software may therefore prove a valuable tool to support control of the HIV epidemic in SA.

Acknowledgements. None.

Author contributions. RRL and RPHP con-
Funding. This study was made possible by the generous support of the American people through the US President's Emergency Plan for AIDS Relief (PEPFAR) through the United States Agency for International Development (USAID) under co-operative agreement no. 674A-12-00015 to the Anova Health Institute. The contents are the responsibility of Anova Health Institute and do not necessarily reflect the views of USAID or the United States Government.

Conflicts of interest. None.

1. Simms I, Gibin M, Petersen J. Location, location, location: What can geographic information science (GIS) offer sexual health research? Sex Transm Infect 2014;90(6):442-443. https://doi. org/10.1136/sextrans-2014-051695

2. Kandwal R, Garg PK, Garg RD. Health GIS and HIV/AIDS studies: Perspective and retrospective. I Biomed Inform 2009;42(4):748-755. https://doi.org/10.1016/j.jbi.2009.04.008

3. Joint United Nations Programme on HIV/AIDS. 90-90-90: An Ambitious Treatment Target to Help End the AIDS Epidemic. Geneva: UNAIDS, 2014.

4. Zulu LC, Kalipeni E, Johannes E. Analyzing spatial clustering and the spatiotemporal nature and trends of HIV/AIDS prevalence using GIS: The case of Malawi, 1994 - 2010. BMC Infect Dis 2014;14:285. https://doi.org/10.1186/1471-2334-14-285

5. Carrel M, Janko M, Mwandagalirwa MK, et al. Changing Carrel M, Janko M, Mwandagalirwa MK, et al. Changing spatial patterns and increasing rurality of HIV prevalence
in the Democratic Republic of the Congo between 2007 and 2013. Health Place 2016;39:79-85. https://doi.org/10.1016/j. 2013. Health Place 2016

6. Tanser F, Barnighausen T, Cooke GS, Newell ML. Localized spatial clustering of HIV infections in a widely disseminated rural South African epidemic. Int J Epidemiol 2009;38(4):10081016. https://doi.org/10.1093/ije/dyp148

7. Messina JP, Emch M, Muwonga J, et al. Spatial and sociobehavioral patterns of HIV prevalence in the Democratic Republic of Congo. Soc Sci Med 2010;71(8):1428-1435. https:// doi.org/10.1016/.jsocscimed.2010.07.025

8. Barankanira E, Molinari N, Niyongabo T, Laurent C. Spatial analysis of HIV infection and associated individual characteristics in Burundi: Indications for effective prevention. BMC Public Health 2016;16:118. https://doi.org/10.1186/ s12889-016-2760-3

9. Goswami ND, Hecker EJ, Vickery C, et al. Geographic information system-based screening for TB, HIV, and syphilis (GIS-THIS): A system-based screening for TB, HIV, and syphilis (GIS-THIS): A
cross-sectional study. PLoS One 2012;7(10):e46029. https://doi. cross-sectional study. PLoS One 201
org/10.1371/journal.pone. 0046029

org/10.1371/journal.pone. 0046029
10. Wand H, Ramjee G. Targeting the hotspots: Investigating Wand $\mathrm{H}$, Ramjee G. Targeting the hotspots: Investigating
spatial and demographic variations in HIV infection in small communities in South Africa. J Int AIDS Soc 2010;13(1):41. https://doi.org/10.1186/1758-2652-13-41

11. Wand H, Whitaker C, Ramjee G. Geoadditive models to assess spatial variation of HIV infections among women in local communities of Durban, South Africa. Int J Health Geogr 2011;10:28. https://doi.org/10.1186/1476-072X-10-28

12. Tanser F, Lesueur D, Solarsh G, Wilkinson D. HIV heterogeneity and proximity of homestead to roads in rural South Africa: An exploration using a geographical information system. Trop Med Int Health 2000;5(1):40-46. https://doi.org/10.1046/j.1365-

3. Cuadros DF, Awad SF, Abu-Raddad LJ. Mapping HIV clustering: A strategy for identifying populations at high risk of HIV infection in sub-Saharan Africa. Int J Health Geogr 2013;12:28. https://doi.org/10.1186/1476-072X-12-28

14. Wabiri N, Shisana O, Zuma K, Freeman J. Assessing the spatial nonstationarity in relationship between local patterns of HIV infections and the covariates in South Africa: A geographically weighted regression analysis. Spat Spatiotemporal Epidemiol 2016;16:88-99. https://doi.org/10.1016/.jste.2015.12.003

15. Cuadros DF, Abu-Raddad LJ. Spatial variability in HIV prevalence declines in several countries in sub-Saharan Africa. Health Place 2014;28:45-49. https://doi.org/10.1016/j. healthplace.2014.03.007

16. Larmarange J, Bendaud V. HIV estimates at second Larmarange $\mathrm{J}$, Bendaud $\mathrm{V}$. HIV estimates at second
subnational level from national population-based surveys. AIDS 2014;28(Suppl 4):S469-S476. https://doi.org/10.1097/
A AIDS 2014;28(Suppl 4)

supervised the project. CJG, TH and RRL managed the data to create the visualisations. All the authors reflected on and interpreted the visualisations. RRL wrote the manuscript, which was critically revised by all the authors. All the authors read and approved the final manuscript.
17. Subnational Estimates Working Group of the HIV Modelling Consortium. Evaluation of geospatial methods to generate subnational HIV prevalence estimates for local level planning. AIDS 2016;30(9):1467-1474. https://doi.org/10.1097/QAD. 0000000000001075

18. Wand H, Ramjee G. Spatial clustering of 'measured' and 'unmeasured' risk factors for HIV infections in hyper-endemic communities in KwaZulu-Natal, South Africa: Results from geoadditive models. AIDS Care 2015;27(11):1375-1381. https:/ doi.org/10.1080/09540121.2015.1096896 
19. Cooke GS, Tanser FC, Barnighausen TW, Newell ML. Population uptake of antiretroviral treatment through primary care in rural South Africa. BMC Public Health 2010;10:585. https://doi. treatment through primary care in

20. Chamla DD, Olu O, Wanyana J, et al. Geographical information system and access to HIV testing, treatment and prevention of mother-to-child transmission in conflict affected Northern Uganda. Confl Health 2007;1:12. https://doi.org/10.1186/1752-1505-1-12

21. Eberhart MG, Yehia BR, Hillier A, et al. Behind the cascade: Analyzing spatial patterns along the HIV care continuum. J Acquir Immune Defic Syndr 2013;64(Suppl 1):S42-S51. https://doi.org/10.1097/ QAI.0b013e3182a90112

22. Goswami ND, Schmitz MM, Sanchez T, et al. Understanding local spatial variation along the care continuum: The potential impact of transportation vulnerability on HIV linkage to care and viral suppression in high-poverty areas, Atlanta, Georgia. J Acquir Immune Defic Syndr 2016;72(1):65-72. https://doi.org/10.1097/QAI.0000000000000914

23. Johnson DC, Feldacker C, Tweya H, Phiri S, Hosseinipour MC. Factors associated with timely initiation of antiretroviral therapy in two HIV clinics in Lilongwe, Malawi. Int J STD AIDS 2013;24(1):42-49. https://doi.org/10.1177/0956462412472312

24. Kloos H, Assefa Y, Adugna A, Mulatu MS, Mariam DH. Utilization of antiretroviral treatment in Ethiopia between February and December 2006: Spatial, temporal, and demographic patterns. Int J Health Geogr 2007;6:45. https://doi.org/10.1186/1476-072X-6-45

25. Yao J, Agadjanian V, Murray AT. Spatial and social inequities in HIV testing utilization in the context of rapid scale-up of HIV/AIDS services in rural Mozambique. Health Place 2014;28:133-141. https:// doi.org/10.1016/j.healthplace.2014.04.007

26. Eberhart MG, Yehia BR, Hillier A, et al. Individual and community factors associated with geographic clusters of poor HIV care retention and poor viral suppression. J Acquir Immune Defic Syndr 2015;69(Suppl 1):S37-S43. https://doi.org/10.1097/QAI.0000000000000587

27. Siedner MJ, Lankowski A, Tsai AC, et al. GPS-measured distance to clinic, but not self-reported transportation factors, are associated with missed HIV clinic visits in rural Uganda. AIDS 2013;27(9):1503-1508. https://doi.org/10.1097/QAD.0b013e32835fd873

28. Zhou YB, Liang S, Wang QX, et al. The geographic distribution patterns of HIV-, HCV- and coinfections among drug users in a national methadone maintenance treatment program in Southwest China. BMC Infect Dis 2014;14:134. https://doi.org/10.1186/1471-2334-14-134

29. Pierce SJ, Miller RL, Morales MM, Forney J. Identifying HIV prevention service needs of African American men who have sex with men: An application of spatial analysis techniques to service American men who have sex with men: An application of spatial analysis techniques to service
planning. J Public Health Manag Pract 2007:Suppl:S72-S79. https://doi.org/10.1097/00124784planning. J Public

30. Martinez AN, Lorvick J, Kral AH. Activity spaces among injection drug users in San Francisco. Int J Drug Policy 2014;25(3):516-524. https://doi.org/10.1016/j.drugpo.2013.11.008

31. Lorway R, Khan S, Chevrier C, et al. Sex work in geographic perspective: A multi-disciplinary approach to mapping and understanding female sex work venues in Southwest China. Glob Public Health 2017;12(5):545-564. https://doi.org/10.1080/17441692.2015.1123748

32. Ferguson AG, Morris CN. Mapping transactional sex on the Northern Corridor highway in Kenya. Health Place 2007;13(2):504-519. https://doi.org/10.1016/j.healthplace.2006.05.009

33. Delaney KP, Kramer MR, Waller LA, Flanders WD, Sullivan PS. Using a geolocation social networking application to calculate the population density of sex-seeking gay men for research and prevention services. J Med Internet Res 2014;16(11):e249. https://doi.org/10.2196/jmir.3523

34. Mahler H, Searle S, Plotkin M, et al. Covering the last kilometer: Using GIS to scale-up voluntary medical male circumcision services in Iringa and Njombe Regions, Tanzania. Glob Health Sci Pract 2015;3(3):503-515. https://doi.org/10.9745/GHSP-D-15-00151

35. Shacham E, Thornton R, Godlonton S, Murphy R, Gilliland J. Geospatial analysis of condom availability and accessibility in urban Malawi. Int J STD AIDS 2016;27(1):44-50. https://doi. availability and accessibility in
org/10.1177/0956462415571373

36. Buehler $\mathrm{CP}$, Blevins $\mathrm{M}$, Ossemane $\mathrm{EB}$, et al. Assessing spatial patterns of HIV knowledge in rural Mozambique using geographic information systems. Trop Med Int Health 2015;20(3):353-364. https:// doi.org/10.1111/tmi. 12437

37. Leibowitz AA, Mendes AC, Desmond K. Public funding of HIV/AIDS prevention, treatment, and support in California. J Acquir Immune Defic Syndr 2011;58(1):e11-e16. https://doi.org/10.1097/ QAI.0b013e31822101c8

38. Bwambale FM, Ssali SN, Byaruhanga S, Kalyango JN, Karamagi CA. Voluntary HIV counselling and testing among men in rural western Uganda: Implications for HIV prevention. BMC Public Health 2008;8:263. https://doi.org/10.1186/1471-2458-8-263
39. Lippman SA, Shade SB, El Ayadi AM, et al. Attrition and opportunities along the HIV care continuum: Findings from a population-based sample, North West Province, South Africa. J Acquir Immune Defic Syndr 2016-73(1):91-99. https//doiorg/10.1097/QAL 0000000000001026

40. Ramirez-Avila L, Nixon K, Noubary F, et al. Routine HIV testing in adolescents and young adults presenting to an outpatient clinic in Durban, South Africa. PLoS One 2012;7(9):e45507. https://doi org/10.1371/journal.pone. 0045507

41. Peltzer K, Matseke G. Determinants of HIV testing among young people aged 18 - 24 years in South Africa. Afr Health Sci 2013;13(4):1012-1020. https://doi.org/10.4314/ahs.v13i4.22

42. Johnson LF, Chiu C, Myer L, et al. Prospects for HIV control in South Africa: A model-based analysis. Glob Health Action 2016;9(1):30314. https://doi.org/10.3402/gha.v9.30314

43. Space-Time Research Pty Ltd. Census 2011: Interactive data in SuperCROSS. SuperCROSS 8.0.2.32. Pretoria: Statistics South Africa, 2011

44. Johnson LF, Dorrington RE, Moolla H. Modelling the impact of HIV in South Africa's provinces: Centre for Infectious Disease Epidemiology and Research working paper. University of Cape Town 2016. http://www.thembisa.org/content/downloadPage/Provinces2016 (accessed 25 July 2017).

45. National Department of Health, South Africa. $\mathrm{NDoH}$ Circular: Implementation of the Universal Test and Treat Strategy for HIV Positive Patients and Differentiated Care for Stable Patients. Pretoria: and Treat St

46. Govindasamy D, Ford N, Kranzer K. Risk factors, barriers and facilitators for linkage to antiretroviral therapy care: A systematic review. AIDS 2012;26(16):2059-2067. https://doi.org/10.1097/QAD. therapy care: A s s
0b013e $3283578 \mathrm{~b} 9 \mathrm{~b}$

47. BBC News Services. Medical motorbike couriers battle HIV. 12 November 2014. http://www.bbc.com/ news/science-environment-30005764 (accessed 18 November 2016).

8. Columbia University Mailman School of Public Health. ICAP launches boat to support HIV services for Tanzania’s Lake Victoria Islands. 20 February 2015. http://icap.columbia.edu/news-events/detail/ icap-launches-boat-to-support-hiv-services-to-tanzanias-lake-victoria-islan (accessed 18 November 2016)

49. Wanyama J, Castelnuovo B, Wandera B, et al. Belief in divine healing can be a barrier to antiretroviral therapy adherence in Uganda. AIDS 2007;21(11):1486-1487. https://doi.org/10.1097/ QAD.0b013e32823ecf7

50. Groh K, Audet CM, Baptista A, et al. Barriers to antiretroviral therapy adherence in rural Mozambique. BMC Public Health 2011;11:650. https://doi.org/10.1186/1471-2458-11-650

51. Root R, Whiteside A. A qualitative study of community home-based care and antiretroviral adherence in Swaziland. J Int AIDS Soc 2013;16:17978. https://doi.org/10.7448/IAS.16.1.17978

52. Kagee A, Remien RH, Berkman A, Hoffman S, Campos L, Swartz L. Structural barriers to ART adherence in southern Africa: Challenges and potential ways forward. Glob Public Health 2011;6(1):83-97. https://doi.org/10.1080/17441691003796387

53. Tuller DM, Bangsberg DR, Senkungu J, Ware NC, Emenyonu N, Weiser SD. Transportation cost impede sustained adherence and access to HAART in a clinic population in southwestern Uganda: $A$ qualitative study. AIDS Behav 2010;14(4):778-784. https://doi.org/10.1007/s10461-009-9533-2

54. Vanmeulebrouk B, Rivett U, Ricketts A, Loudon M. Open source GIS for HIV/AIDS management. In J Health Geogr 2008; 7:53. https://doi.org/10.1186/1476-072X-7-53

55. Kamadjeu R, Tolentino $\mathrm{H}$. Web-based public health geographic information systems for resourcesconstrained environment using scalable vector graphics technology: A proof of concept applied to the expanded program on immunization data. Int J Health Geogr 2006;5:24. https://doi.org/10.1186/1476$072 \mathrm{X}-5-24$

56. Tanser FC, le Sueur D. The application of geographical information systems to important public health problems in Africa. Int J Health Geogr 2002;1(1):4. https://doi.org/10.1186/1476-072X-1-4

57. Tanser FC. The application of GIS technology to equitably distribute fieldworker workload in a large rural South African health survey. Trop Med Int Health 2002;7(1):80-90. https://doi.org/10.1046 j.1365-3156.2002.00825.x

58. Busgeeth K, Rivett $\mathrm{U}$. The use of a spatial information system in the management of HIV/AIDS in South Africa. Int J Health Geogr 2004;3(1):13. https://doi.org/10.1186/1476-072X-3-13

Accepted 14 August 2017 